Artículo

Los textos publicados son responsabilidad exclusiva de sus autores

\title{
La incidencia de la condición sociodemográfica en el rendimiento académico de los estudiantes del Profesorado de Enseñanza Media en Lengua y Literatura
}

The incidence of the sociodemographic condition in the academic performance of the students of the Teaching Staff of Secondary Education in Language and Literature

\author{
Dora Reyes Santa Cruz \\ Maestría en Investigación \\ Universidad de San Carlos de Guatemala \\ https://orcid.org/0000-0003-2445-9281 \\ doreycruzencf19@gmail.com
}

\section{Referencia}

Santa Cruz, D. R. (2021). La incidencia de la condición sociodemográfica en el rendimiento académico de los estudiantes del Profesorado de Enseñanza Media en Lengua y Literatura. Revista Guatemalteca de Educación Superior, 4(1), 12-22.

DOI: https://doi.org/10.46954/revistages.v4i1.50

Recibido: 15/11/2020

Aceptado: 16/01/2021

\section{Resumen}

Este estudio tuvo como objetivo establecer la incidencia de la condición sociodemográfica en el rendimiento académico de los estudiantes del Profesorado de Enseñanza Media en Lengua y Literatura, de la Facultad de Humanidades, Sede Central, Plan Sábado. En la investigación se aplicó el diseño no experimental, de enfoque cuantitativo y alcance descriptivo. Se utilizó la base de datos de punteos del curso de Gramática Descriptiva II y se realizó una encuesta a 95 estudiantes del quinto ciclo.

Los resultados evidenciaron que el $84.2 \%$ de estudiantes residen en el municipio de Guatemala, mientras que el $15.8 \%$ se 
distribuye entre los departamentos de Escuintla, Sacatepéquez y Chimaltenango. El $89 \%$ de los estudiantes son mujeres. Además, el rango de edad de los estudiantes está entre 20 y 54 años. En relación con el rendimiento académico los estudiantes presentan un nivel regular, puesto que la media en la base de datos de Gramática Descriptiva II es de 66 puntos. Por otra parte, se observa que los cursos de literatura son los que más han repetido los estudiantes.

El nivel sociodemográfico no incide de manera significativa en el rendimiento académico de los estudiantes. En cuanto al estado civil, hay casados, solteros y divorciados, en donde el $71.6 \%$ es de solteros. El $28.4 \%$ está en condición de desempleo, y se infiere que este factor socioeconómico afecta el rendimiento académico, porque en determinado momento no poseen los recursos económicos necesarios para cubrir lo que se considera esencial, verbigracia, muchos de ellos tuvieron dificultad para mantener un adecuado servicio de internet al recibir clases a distancia, por la situación de la pandemia covid-19.

\section{Abstract}

This study aimed to establish the incidence of sociodemographic condition in the academic performance of the students of the Teaching Staff of Secondary Education in Language and Literature, of the Faculty of Humanities, Headquarters, Saturday Plan. In the research, the non-experimental design, with a quantitative approach and descriptive scope, was applied. The scores database from the Descriptive Grammar II course was used and a survey was conducted with 95 fifth-cycle students.

The results showed that $84.2 \%$ of students reside in the municipality of Guatemala, while $15.8 \%$ are distributed among the departments of Escuintla, Sacatepéquez and Chimaltenango. $89 \%$ of the students are women. Furthermore, the age range of the students is between 20 and 54 years old. In relation to academic performance, students present a regular level, since the mean in the Descriptive Grammar II database is 66 points. On the other hand, it is observed that literature courses are the ones that students have repeated the most.

\section{Palabras clave:}

sociodemografía, rendimiento académico, condición socioeconómica. 


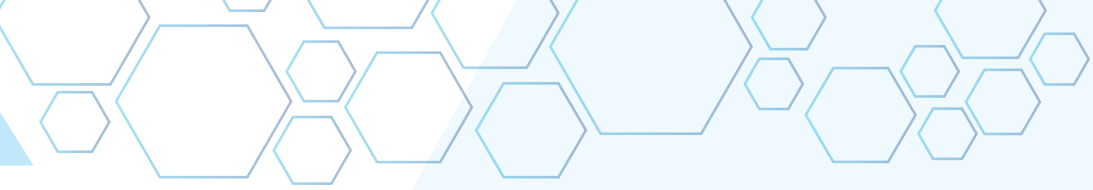

\section{Keywords:}

sociodemography, academic performance, socioeconomic status.
The sociodemographic level does not significantly affect the academic performance of students. Regarding marital status, there are married, single and divorced persons, where $71.6 \%$ are single. $28.4 \%$ are unemployed, and it is inferred that this socioeconomic factor affects academic performance, because at a certain moment they do not have the necessary economic resources to cover what is considered essential, for example, many of them had difficulty maintaining an adequate Internet service when receiving distance classes, due to the situation of the covid-19 pandemic.

\section{Introducción}

Se consideró que en los aspectos sociodemográficos podría encontrarse el origen de los resultados del rendimiento académico de los estudiantes. Por lo que en esta investigación se propuso establecer la incidencia de la condición sociodemográfica y el rendimiento académico de estudiantes del Profesorado de Enseñanza Media en Lengua y Literatura, FAHUSAC, Sede Central, Plan Sábado, en el curso de Gramática Descriptiva II.

Las características generales sociodemográficas se refieren al tamaño de un grupo poblacional y los rasgos que dan forma a la identidad de los integrantes de esta agrupación, que marcarán las diferencias y similitudes entre los individuos que los identifican - agrupan en un momento determinado, según lo afirma Ramírez (2013). Por esto se analizó la edad, el sexo, el estado civil, su procedencia geográfica y su condición socioeconómica y aspectos socioeconómicos que se consideraron las principales variables clasificatorias para este estudio.

Del rendimiento académico, opinan Castro, et al (2009) que existen variados factores que influyen en el rendimiento académico como elementos psicosociales, socioeconómicos, aspectos sociodemográficos, las metodologías de enseñanza, coeficiente Intelectual, estilos de aprendizaje, motivación. Además, indica Castro que el estilo de aprendizaje se refiere al hecho de que cada persona utiliza su propio método para aprender, sus propios hábitos de aprendizaje (por ser adultos) y que la calificación o indicador de logro que se observa a través de las evaluaciones, solo evidencia el nivel de conocimientos 
académicos adquiridos en un determinado momento del proceso educativo.

Se pudo establecer que la condición sociodemográfica de los estudiantes encuestados, de acuerdo con los resultados, la mayoría reside en el departamento de Guatemala; son pocos los que se movilizan de los departamentos a recibir clases a la ciudad capital. Se comprobó que la mayoría de estudiantes son mujeres y solamente el $10.5 \%$ son hombres; los solteros conforman la tercera parte del grupo en estudio. Además, en el momento que se realizó la encuesta, el $24 \%$ no tenía un empleo; los estudiantes que estaban trabajando, solo el $16.8 \%$ se desempeñaba en educación media. Los resultados finales que reveló la base de datos del curso de Gramática Descriptiva Il demostraron tener un promedio regular.

\section{Materiales y métodos}

El diseño aplicado en esta investigación fue no experimental. Los estudios no experimentales se realizan sin la manipulación deliberada de variables y solo se observan los fenómenos en su ambiente natural. El enfoque de este estudio fue cuantitativo; el alcance fue descriptivo, pues se buscó especificar propiedades y características importantes del grupo o población en estudio. El diseño de la muestra no responde a ningún procedimiento estadístico, pues se tomó a los estudiantes que completaron el curso de Gramática descriptiva II.

El criterio único de inclusión se basó en que fueran estudiantes legalmente inscritos en el quinto ciclo y en el curso de Gramática Descriptiva II, en el PEM de Lengua y Literatura, del Plan Sábado. Eran 105 estudiantes, de los cuales participaron 95 de forma voluntaria. La encuesta se estructuró con 23 cuestiones divididas en los siguientes apartados: procedencia geográfica, información personal, condición económica, rendimiento académico y comentarios sobre la carrera. 


\section{Resultados}

La encuesta demostró que los estudiantes para ir a estudiar a la Universidad de San Carlos, viajan de los departamentos de Chimaltenango, Sacatepéquez y Escuintla en un 20\% y de Guatemala en un $80 \%$. El $72 \%$ de dicha población viven en el área urbana, el 24\% en el área rural y el 4\%, en el área suburbana; por lo tanto, no representa un factor significativo que incida en el rendimiento académico, pues la mayoría se moviliza en el perímetro de la Ciudad. Otros hallazgos, la preparación en educación media que los encuestados tienen es diversa, el $42 \%$ dijreron tener título de maestro o bachiller en educación y el 68\% aseveraron tener títulos de otras carreras, como secretariados, peritos y diversas especialidades de bachilleratos.

Se encontró que en la edad de los estudiantes existía una gran diferencia, ya que el rango va de 20 hasta 54 años de edad. Otro aspecto que se observó fue la variable sexo, pues hay un número mayoritario de mujeres correspondiente al $89.5 \%$ y solamente el $10.5 \%$ es de hombres. Además, el estado civil predominante en este grupo fue de solteros con $61 \%$ y luego sigue el de casados con $27 \%$ y el resto es de divorciados y los que están en unión de hecho.

Figura No. 1 Ingresos económicos familiares

¿A cuánto ascienden sus ingresos familiares?

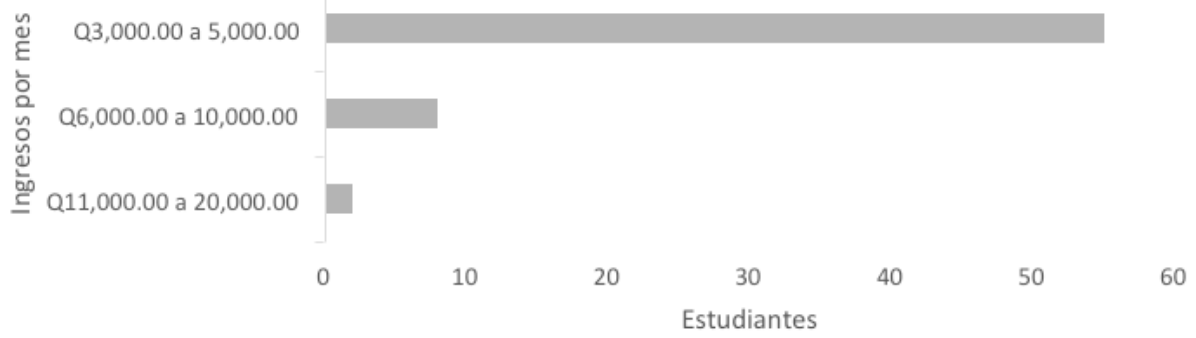

Fuente: información de encuesta aplicada a estudiantes de la carrera de Profesorado de Enseñanza Media en Lengua y Literatura (2020). 
Al preguntar sobre los ingresos económicos familiares, el 82\% de los encuestados refirieron que se encontraban entre tres a cinco mil quetzales mensuales; un $16.9 \%$ afirmaron que sus ingresos familiares estaban entre seis y diez mil o más quetzales al mes.

Tabla No. 1 Tipo de trabajo que realiza

\begin{tabular}{lcc}
\hline Tipo de trabajo & Frecuencia & Porcentaje \\
\hline Contabilidad & 1 & 1.1 \\
\hline Dependiente & 2 & 2.1 \\
\hline Emprendimiento & 2 & 2.1 \\
\hline Maestro de educación preprimaria & 8 & 8.4 \\
\hline Maestro de educación primaria & 23 & 24.2 \\
\hline Profesor de nivel medio & 16 & 16.8 \\
\hline Secretarial & 6 & 6.3 \\
\hline Otro & 14 & 14.7 \\
\hline No trabaja & 23 & 24.2 \\
\hline Total & 95 & 100.0 \\
\hline
\end{tabular}

Fuente: información de encuesta aplicada a estudiantes de la carrera de Profesorado de Enseñanza Media en Lengua y Literatura (2020).

Los sectores donde trabaja la mayoría de estudiantes no tienen relación con la educación superior que están recibiendo, únicamente el $16.8 \%$ de ellos trabaja en la docencia como Profesores de Enseñanza Media. El 24.2\%, se desempeña como maestro de educación primaria y el $8.4 \%$, labora en la preprimaria. El $56.6 \%$ ejerce diferentes profesiones u oficios o no trabaja. 
Figura No. 2 Rendimiento académico que indicaron los estudiantes
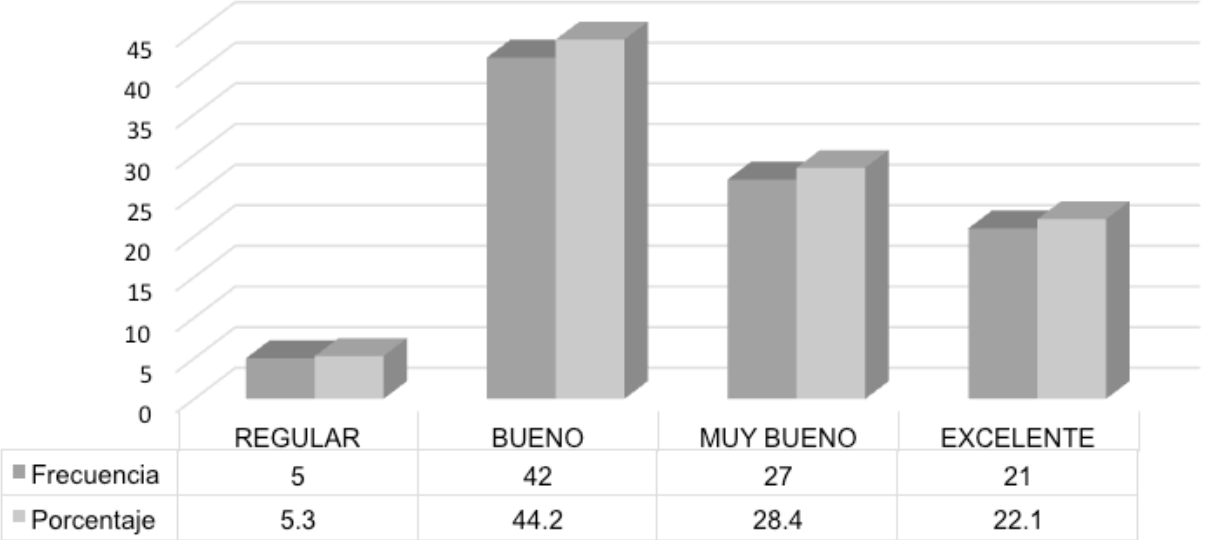

Fuente: información de encuesta aplicada a estudiantes de la carrera de Profesorado de Enseñanza Media en Lengua y Literatura (2020).

Al preguntar a los encuestados sobre cómo perciben su rendimiento académico en los ciclos ya cursados, el $44.2 \%$ indicaron que es bueno, el $28.4 \%$ perciben tener un rendimiento muy bueno y el $22.1 \%$ señalaron tener un rendimiento excelente.

\section{Discusión}

La condición sociodemográfica: la condición civil, la edad, el sexo y su procedencia geográfica no refleja incidencia en un mejor rendimiento académico. En el caso de los estudiantes que residen fuera del departamento de Guatemala, que se esperaba fueran los más afectados, no lo fue, es más, les favoreció el no tener que movilizarse debido a la pandemia, ya que sus estudios los realizaron a distancia.

El hallazgo en la condición socioeconómica fue más evidenciable, pues el $71.6 \%$ de estudiantes trabajan y el $28.4 \%$ está en condición de desempleado; este factor sí puede determinar el rendimiento académico, porque en determinado momento no poseen los recursos económicos para cubrir sus necesidades básicas. Las respuestas relacionadas a los ingresos familiares fueron respondidas por el $60 \%$ de los encuestados. El hallazgo 
que evidenció la condición socioeconómica fue que el $82 \%$ tiene ingresos entre tres mil y cinco mil quetzales al mes, significa que se encuentran en condiciones de pobreza.

Entre los factores que se asocian al rendimiento académico está la pobreza. Marina, Gerónimo y Pérez (2017) opinan que los vínculos entre la existencia de la pobreza y la educación son limitantes, porque una persona en condición de pobreza tiene una menor probabilidad de acceder a niveles más altos en la educación superior, y esto, frecuentemente, le obliga a vivir en pobreza. En la investigación realizada por Bollmann (2013), se clasifica a la sociedad guatemalteca por los ingresos y su capacidad de compra. En el caso de los estudiantes estarían en el grupo D1 y D2 que forman el nivel más bajo, con un ingreso promedio menor de 07,200.00 al mes.

Los trabajos que ejercen la mayoría de estudiantes no tienen relación con la carrera que estudian, Profesorado de Enseñanza Media en Lengua y Literatura. Únicamente el 16\% de ellos trabaja educación media, el $23 \%$ en educación primaria y el $8 \%$ en educación preprimaria. Como se puede observar, la condición laboral tiene poca relación con sus estudios. Los datos de la edad y el sexo coincidieron con el Informe Estadístico Estudiantil 2019, Departamento de Registro y Estadística (2019), que muestran que el $43 \%$ de estudiantes entre 20 y 27 años de edad, el $37 \%$ entre 28 y 41 años y el $20 \%$ de 42 a 55 años. El $89.5 \%$ de estudiantes son mujeres y solamente el $10.5 \%$ de los estudiantes son hombres.

El rendimiento académico que refleja la base de datos del curso de Gramática Descriptiva II, está en un promedio de 66 puntos, lo que la Universidad de San Carlos clasifica como regular, pues se aprueba con 61 puntos. Se considera que los estudiantes del Profesorado en Enseñanza Media en Lengua y Literatura del Plan Sábado de la USAC, a pesar de los factores socioeconómicos y ser estudiantes trabajadores, continúan con su preparación académica.

García-Allen (2019) describe la motivación positiva como la que el sujeto inicia o mantiene apegado a una conducta por la recompensa positiva, ya sea externa o interna (por el placer 
de la actividad) y la negativa se refiere cuando una persona se mantiene adherida a una conducta para evitar una consecuencia desagradable, tanto externa (castigo, humillación, etc.) o interna (evitar la sensación de frustración o fracaso). Los estudiantes de fin de semana, además de estar motivados, deben manejar con mucha madurez la autorregulación.

Alonso-Tapia (2014) indica que la autorregulación es una competencia que permite a los alumnos activar las estrategias de aprendizaje, que son necesarias para alcanzar los objetivos establecidos. Asimismo, es el proceso formado por pensamientos autogenerados, emociones y acciones que están planificadas y adaptadas cíclicamente para lograr la obtención de los objetivos personales. La motivación y la autorregulación son factores que pueden afectar o favorecer al rendimiento académico. En el caso del grupo en estudio, lo han manejado a su favor.

Se afirma que los aspectos sociodemográficos no incidieron significativamente en los resultados académicos de los estudiantes del Profesorado de Enseñanza Media en Lengua y Literatura del Plan Sábado de la Universidad de San Carlos de Guatemala. Sin embargo, la condición socioeconómica sí evidencia la relación que existe entre desear y realizar, porque muchos estudiantes desean superarse académicamente, pero pocos pueden hacer realidad ese deseo.

Como resultado de observar esta situación, se sugiere crear un Programa de Becas que beneficie, en especial, a los estudiantes trabajadores, pues ellos son los que más necesitan este tipo de ayuda. Ya que los ingresos, según los hallazgos del estudio, demuestran que más del $71.6 \%$ de estudiantes tienen ingresos que los clasifica como personas con nivel de ingresos bajos, es decir, en pobreza.

\section{Agradecimientos}

A la doctora Patricia Luz Mazariegos Romero, por la asesoría brindada, su persistencia en todo momento y sus consejos. A la maestra Dunia Marcela Marroquín Miranda, por las revisiones. A las licenciadas Ruth Noemí Vicente Gómez y Elvia Alvarado de Yantuche, por su apoyo incondicional y la revisión de este artículo. 


\section{Referencias}

Alonso- Tapia, J. (2014). Teorías de autorregulación educativa: una comparación y reflexión teórica. Psicología Educativa, 20(1), 11-22. https://doi.org/10.1016/j.pse.2014.05.002

Bollmann, C. (29 de 11 de 2013). Guatemala: presenta estudio sobre niveles socioeconómicos. Empresas y management. Recuperado el 26 de octubre de 2020, de https://www. estrategiaynegocios.net/lasclavesdeldia/562566-330/ guatemala-presentan-estudio-sobre-nivelessocioeconomicos

Castro, M., Aguirre, M., \& Adasme, A. (abril de 2009). Factores que inciden en el rendimiento escolar en Chile. Centro de Estudios de Opinión Ciudadana, 2(4). Recuperado el noviembre de 2020, de https://es.slideshare.net/ ManuelMiranda4/factores-que-inciden-en-el-rendimientoescolar-2009

García-Allen, J. (2020). Tipos de motivación: las 8 fuentes motivacionales. Psicología y Mente. Recuperado el noviembre de 2020, de https://psicologiaymente.com/ psicologia/tipos-de-motivacion

Marina, J., Gerónimo, V., \& Pérez, J. (13 de 12 de 2017). Efectos de la pobreza y de los factores sociodemográficos en la educación. Nova Scientia, 10(20), 539--568. https://doi. org/10.21640/ns.v10i20.1159

Ramírez, P. O. (septiembre de 2013). Sociodemografía ciencia - teoría. Scribd Acquires SlideShare. Recuperado el noviembre de 2020, de https://es.slideshare.net/ pedrito236/sociodemografia-ciencia-o-teoria

Universidad de San Carlos de Guatemala, Dirección General de Administración. (2019). Universidad de San Carlos de Guatemala, Dirección General de Administración Departamento de Registro y Estadística 2019, Informe Estadístico Estudiantil USAC 2019, Informe Ejecutivo. Universidad de San Carlos de Guatemala, Guatemala. Guatemala: USAC. Recuperado el noviembre de 2020, de https://registro.usac.edu.gt/estadisticas/data/INFORME_ ESTADISTICO_ESTUDIANTIL_2019.pdf 


\section{Sobre la autora}

\section{Dora Reyes Santa Cruz}

Es licenciada en Letras, graduada del Departamento de Letras, Facultad de Humanidades de la Universidad de San Carlos de Guatemala, trabaja como docente en el Departamento de Letras, Facultad de Humanidades y docente en el Área Básica de la Facultad de Odontología, Universidad de San Carlos de Guatemala.

Copyright (c) Dora Reyes Santa Cruz

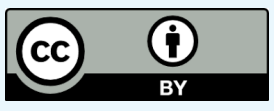

Este texto está protegido por una licencia CreativeCommons 4.0.

Usted es libre para compartir, copiar y redistribuir el material en cualquier medio o formato y adaptar el documento, remezclar, transformar y crear a partir del material para cualquier propósito, incluso comercialmente, siempre que cumpla la condición de atribución: usted debe reconocer el crédito de una obra de manera adecuada, proporcionar un enlace a la licencia, e indicar si se han realizado cambios. Puede hacerlo en cualquier forma razonable, pero no de forma tal que sugiera que tiene el apoyo del licenciante o lo recibe por el uso que hace. 